\title{
Radial collapse of carbon nanotubes for conductivity optimized polymer composites
}

\author{
Félix Balima ${ }^{\mathrm{a}, \mathrm{b}}$, Sylvie Le Floch ${ }^{\mathrm{a}}$, Christophe Adessi ${ }^{\mathrm{a}}$, Tiago F.T. \\ Cerqueira $^{\mathrm{a}, \mathrm{c}}$, Nicholas Blanchard ${ }^{\mathrm{a}}$, Raúl Arenal ${ }^{\mathrm{d}, \mathrm{e}}$, Annie Brûlet ${ }^{\mathrm{f}}$, Miguel \\ A.L. Marques ${ }^{\mathrm{g}}$, Silvana Botti ${ }^{\mathrm{h}}$, Alfonso San-Miguel ${ }^{\mathrm{a}, *}$ \\ ${ }^{a}$ Université de Lyon, F-69000 Lyon, France and Institut Lumière Matière, CNRS, UMR \\ 5306, Universitè Lyon 1, F-69622 Villeurbanne, France \\ ${ }^{b}$ Institut de Chimie de la Matière Condensée de Bordeaux, UPR9048 CNRS, Pessac, \\ France \\ ${ }^{c}$ Institut für Festkörpertheorie und -optik, Friedrich-Schiller-Universität Jena, \\ Max-Wien-Platz 1, 07743 Jena, Germany \\ ${ }^{d}$ Laboratorio de Microscopias Avanzadas(LMA) - Instituto de Nanociencia de Aragon \\ (INA), U. Zaragoza, 50018 Zaragoza, Spain \\ ${ }^{e}$ Fundacion ARAID, 50018 Zaragoza, Spain \\ ${ }^{f}$ Laboratoire Léon Brillouin, UMR12 CEA-CNRS, CEA-Saclay, Gif-sur-Yvette, France \\ ${ }^{g}$ Institut für Physik, Martin-Luther-Universität Halle-Wittenberg, D-06099 Halle, \\ Germany \\ ${ }^{h}$ Institut für Festkörpertheorie und-optik, Friedrich-Schiller Universität Jena, \\ Max-Wien-Platz 1, 07ry3 Jena, Germany
}

\begin{abstract}
The optimization of the electronic conduction of carbon nanotube polymer composites is studied by tuning the radial geometry of the carbon nanotubes in a compression cycle. We have investigated the structural evolution of multi-walled carbon nanotubes in a polyamide matrix as a function of applied high pressure. Combining high resolution electron microscopy and small angle neutron scattering experiments, we conclude that the nanotube radial cross-section is irreversibly deformed following applied pressures up to 5 GPa. Studying highly percolated composites we observe that the sample resistivity drastically decreases with pressure up to about $2 \mathrm{GPa}$ with no further change up to the maximum $5 \mathrm{GPa}$ applied pressure. An important hysteresis is observed upon decompression which leads to an enhanced electrical con-
\end{abstract}

\footnotetext{
* Corresponding author

Email address: alfonso.san-miguel@univ-lyon1.fr (Alfonso San-Miguel)
} 
ductivity of the composite in all the studied compression cycles with maximum pressures ranging from 1 to $5 \mathrm{GPa}$. Modelling the radial collapse of single-walled carbon nanotubes shows that the modified radial geometry can considerably improve the electronic transport properties in contacted carbon nanotube junctions. Our results open opportunities for engineering nanotube composites by controlling the radial collapse.

\section{Introduction}

The exceptionally high elastic modulus and tensile strength of carbon nanotubes $(\mathrm{CNT})$ is driving their development as reinforcing agents in next generation composite materials $[1,2,3]$. These properties, together with their electrical and thermal conductance, have been decisive for an emerging industrial interest in CNT in low weight reinforced composites, conductive polymers or advanced nanocomposites with multi-functional features. Nevertheless several challenges need to be tackled before CNT realize their full potential; such as poor dispersion or alignment[4], which together with higher production costs presently prevent CNT to fully compete with carbon fibers. Nevertheless, CNT composites remain attractive as they exhibit very low electric percolation thresholds[5]. Further developments of CNT-based composites, taking advantage of CNT exceptional physical properties, are in all cases needed from synthesis methods and scaling to system design.

Most of the physical properties of CNT rely on their particular geometry: an atomic carbon hollow cylinder with a very high aspect ratio. The circular cross-section constitutes an important characteristic feature, which is determinant for many CNT properties.

The modification of the CNT cross-section geometry constitutes a parameter which up to now has not attracted much attention in the design of CNT composite materials. It is nevertheless known that the radial crosssection adopted by CNT depends on their geometrical parameters (diameter and number of walls) and can be very far from circular[11]. The collapsed structure, also called dog-bone type has been observed in a number of largediameter single-walled (SWCNT), double-walled (DWCNT) and few-walled (FWCNT) carbon nanotubes at ambient pressure[6, 12, 9, 7, 8, 13, 14]. Some of these observations are summarized in Fig 1. For very large diameter FWCNT, the collapsed geometry has been proposed as an analogue for graphene nanoribbon structures[15]. The collapsed structure is stabilized 


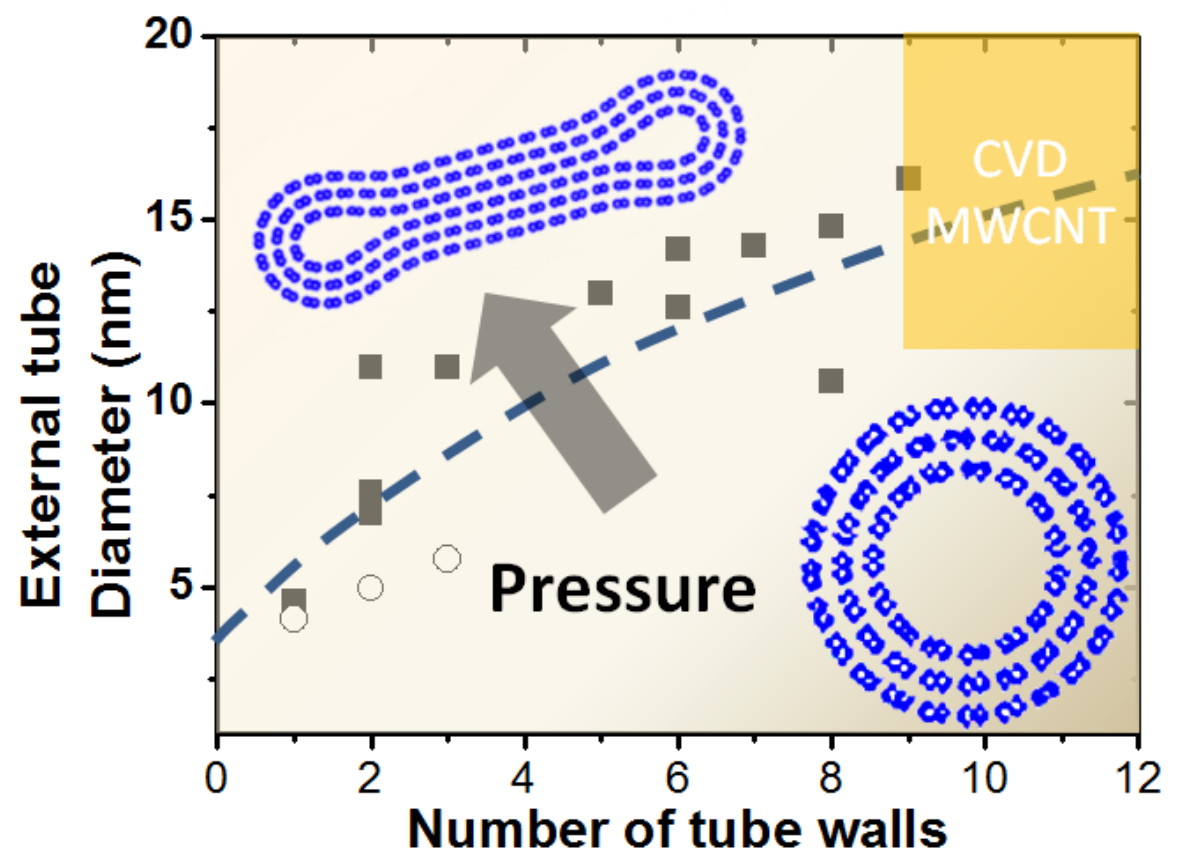

Figure 1: CNT with collapsed geometry at ambient conditions. Each filled square represent a TEM observation of a collapsed CNT with a given number of walls and tube-diameter $[6$, $7,8,9]$. The open circles correspond to values calculated by atomistic modeling[10]. The discontinous line is a guide for the eye. Tubes in the upper region of the figure (above the line) should then be expected to be naturally collapsed at ambient conditions while those in the lower part could keep a circular cross-section at ambient conditions. In the right upper corner is shown the region where lie the CVD MWCNTs used in this study. The arrow shows the expected effect of pressure (see text) 
through the additional van der Waals interactions provided by the inter-wall interaction which compensates for the involved elastic energies[16]. A number of precautions should be taken when considering published observations of collapsed tubes, such as channelling effects in TEM measurements that can contribute to the tube collapse,[12] as well as substrate interactions [7, 17] that can modify the tube cross-section. The existence of a wide region of metastability of collapsed/non-collapsed structures has been predicted[10]. Atomistic calculations[18, 10] (also included in Fig. 1) seem to confirm the experimental trend indicating that at ambient pressure the collapsed tube geometry is governed by the number of walls and the radius of the tube.

Radial collapse of carbon nanotubes can be also obtained by applying pressure. In SWCNT the pressure induced radial collapse has been both theoretically predicted $[19,16,20,21]$ and experimentally observed $[22,23,24]$. There exists as well a number of predictions of pressure induced collapse in MWCNT[25, 26, 27].

There have been a few predictions on the electronic structure evolution of SWCNT subjected to radial deformations, [28, 29, 30, 31, 32, 33, 34, 35]: they basically show that both metal-semiconductor and semiconductor-metal transitions are possible, depending on the tube chirality and on the degree of deformation. There have been some experimental confirmations of both a semiconductor to metal transition in SWCNT[36] and of a metalsemiconductor transition in DWCNT[37] upon radial collapse.

There have been many efforts on the optimization of polymer composites[38] and in particular the conductivity of polyamide-MWCNT nanocomposites $[39,5,40]$. In this work we show that the electrical conductivity of polyamideMWCNT composites can be markedly improved through a high pressure treatment by surpassing the usual pressure limits of hot pressing or extrusion methods. We provide direct evidence of the pressure induced collapse of MWCNT in the compressive process and theoretical understanding of the relation between the geometrical changes of the nanotubes and the modification of the conductivity of the percolated network. Our work shows that the very high compression of MWCNT polymer composites can provide then an additional mechanism for electrical conductivity optimization, beyond the system consolidation. 


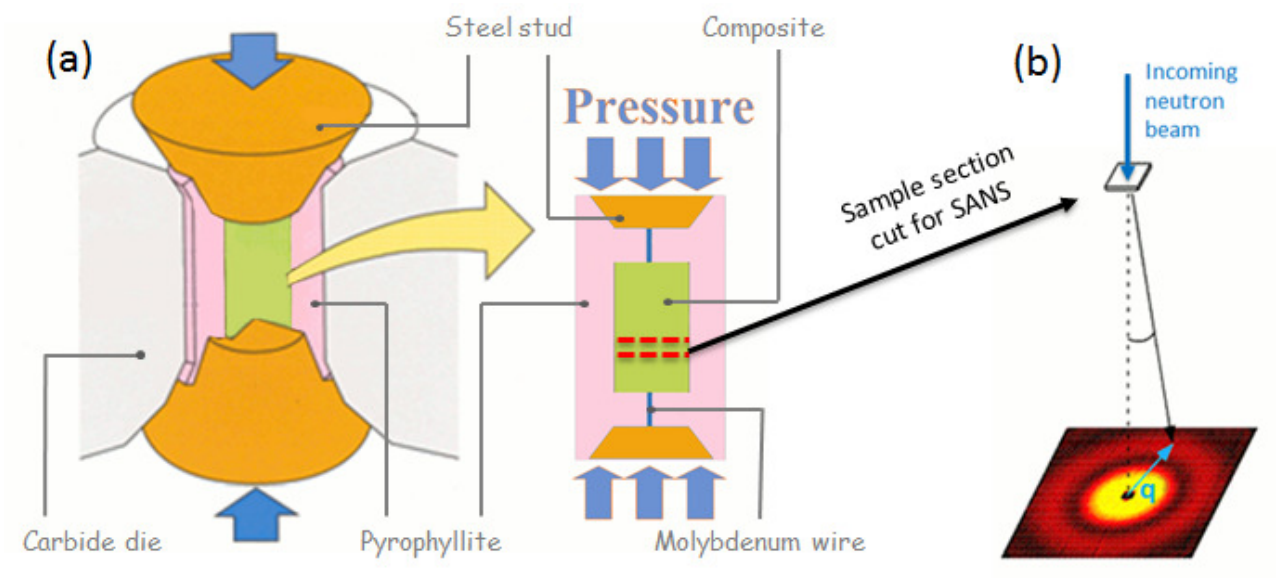

Figure 2: (a) Experimental set-up used for the in situ characterization of the electrical resistivity evolution of the composite with pressure. The principle of our belt-type apparatus is shown in which the sample is confined between 2 steel studs which are pressed by two symmetric carbide anvils (not shown) and a carbide die (the belt). A pyrophylite cylinder plays the role of pressure transmitting medium. The zoom shows the molybdenum wires used to drive the current to the composite samples for the resistivity measurements. The doted lines show a section of the sample cut perpendicularly to the compression axis after the pressure cycle for the different ex situ probes. (b) In the right part is shown how the high pressure cycled samples where oriented with respect to the neutron beam for SANS experiments. The scattering vector $q$ is shown on the 2-dimensional detector 


\section{Experimental and Numerical Methods}

\subsection{Experimental Methods}

Samples were industrial batches of CVD grown MWCNT (Graphistrength (R) 100 ) dispersed in a polyamide PA12 matrix by extrusion at high temperature (Arkema C M1-20 mixture). They consisted of black solid pellets containing perfectly dispersed MWCNT at a concentration of $20 \%$ by weight. The nominal moisture content is of $0.10-0.15 \%$ by weight.Information of the physical characteristics of the associated nanotubes and composites can be found in different works $[41,42]$. The high CNT loading (20\% by weight) assures a concentration above the detection limits of the different characterization probes used in this study and more particularly in the case of small angle neutron scattering. The same composition is kept for all the experimental techniques used in the study. The typical diameter distribution of the MWCNT is shown in the supplementary material. The geometries of MWCNT show a broad distribution of sizes, with most tubes having 10-15 walls with an outer mean diameter of $12 \mathrm{~nm}$. As seen in Fig. 1, some of these tubes may lie in a geometrical domain where modified radial crosssections exist at ambient conditions. We will come back to this point when presenting the high-resolution transmission electron microscopy (HRTEM) measurements. This is promising for industrial protocols as applying moderate pressure could easily modify the MWCNT cross-section.

Using a Belt pressure apparatus[43], we measured the resistance of masterbatches in situ under pressure. The cylindrical shape samples were placed in a pyrophillite cell which is electrically insulating (see Fig. 2). This pyrophyllite cell was machined for each sample to match its dimensions which could range from 3 to $3.5 \mathrm{~mm}$ in diameter and from 5 to $6 \mathrm{~mm}$ in height. Molybdenum wires ( $1 \mathrm{~mm}$ in diameter) were brought out of the pyrophillite cell in order to assure the electrical contact between the end surface of the sample covered with an aluminium foil and the tungsten carbide conical punch. The sample was pressed at room temperature up to a maximum pressure. For each type of masterbatch, several experiments were performed reaching different maximum pressures of $0,1,2,3,4$ and 5 GPa. The electrical resistance measurements were made in situ using a Keithley 2010 multimeter. Following the displacement sensor evolution which allowed the sample length to be monitored, we calculated the resistivity knowing the sample dimensions at the beginning, at the maximum pressure and after decompres-

sion. The electrical resistance was also measured out of the high pressure 
device before and after the pressure cycle. The electrodes were placed at both end surfaces of the cylindrical shape sample. Copper sheets were attached to each end surfaces to ensure good contact of the sample surface with the electrode.

To obtain more insight on the processes involved in the in situ resistivity experiments, the samples recovered after the pressure cycle where examined by Raman spectroscopy, transmission electron microscopy (TEM), high-resolution TEM (HRTEM) and small angle neutron scattering (SANS). Resistivity measurements were also carried out on the recovered samples.For the different experiments, the samples were cut in slices perpendicular to the compression axis of different thickness depending on the experimental probe as illustrated in Fig NEW.

The transmission electron microscopy (TEM) samples were slices of 50 nm thickness cut using the cold microtomy technique. High resolution TEM (HRTEM) was performed with an FEI Titan-Cube microscope working at $80 \mathrm{kV}$, equipped with a Cs corrected objective lens (CETCOR from CEOS $\mathrm{GmbH}$ ). Transmission electron conventional images were obtained using a TOPCON EM-002B microscope $(120 \mathrm{kV})$.

SANS experiments were carried out on the PAXY beamline at the Laboratoire Léon Brillouin (LLB, Saclay, France) using a He multi-detector. Neutrons with a wavelength of $6 \AA$ and two sample-to-detector distances of 3065 and $6765 \mathrm{~mm}$ were used, resulting in a q range from $5.7 \times 10^{-3}$ to $1.5 \times 10^{-1} \AA^{1}$. The samples used in the SANS experiment were taken from the starting composite masterbatches and from those submitted to the pressure/decompression cycle in the Belt device. They were cut to obtain thin samples with thickness ranging from 1 to $2 \mathrm{~mm}$ (see Fig. 2.b). The sample cross-section was around $3.5 \mathrm{~mm}$ in diameter. We then used a $3 \mathrm{~mm}$ diameter beam size. The raw scattering intensities were corrected to account for sample transmission as well as sample thickness following classical SANS data analysis procedures[44]. Differential cross sections per unit sample volume were obtained using plexiglas sheet as normalized standard and direct determination of incident neutron beam flux method[44]. Integrated data were obtained from the raw data using the LLB in-house software PAsiNET[45]. SANS data were fitted using SasView 3.0.0 software $^{1}$

Raman Spectrometry characterizations were done on backscattering ge-

\footnotetext{
${ }^{1}$ http://www.sasview.org/
} 
ometry using a $514 \mathrm{~nm}$ excitation wavelength on a home-made spectrometer. See Reference [46] for more details.

Raman spectroscopy and conventional TEM images did not show significant changes when comparing the signal before and after the pressure cycle up to $5 \mathrm{GPa}$. The TEM images showed a good dispersion of tubes and numerous possibilities for the tubes to be in contact, forming a well percolated network. For these two techniques, results are included in the supplementary material.

\subsection{Modeling Methods}

We have explored numerically the effect of the tube collapse on a system of two SWCNT. The geometry of the collapsed tubes was calculated using quasi-static DFTB calculations on SWCNT bundles using the same method as in Ref. [21].

The resistance at the Fermi level of the structures is computed using a Landauer-Büttiker Formalism. We start with an ab initio calculation of the system using the SIESTA code [47]. The Hamiltonian obtained from SIESTA is then used to compute the transmission using an electronic transport code [48]. The conductance (and so the resistance) is derived from the relation (see reference [49]):

$$
G\left(E_{f}\right)=\frac{2 e^{2}}{h} \int T(E)\left[-\frac{d}{d E} \frac{1}{e^{\left(E-E_{f}\right) /\left(k_{B} T\right)}+1}\right] d E,
$$

where $T(E)$ is the transmission, $E_{f}$ the Fermi level, $k_{B}$ the Boltzmann constant and $T$ the temperature. In this work we have used ambient temperature i.e. $300 \mathrm{~K}$.

We also studied the effect of a junction between a pristine section and a collapsed one in a same tube. The case of a $(12,0)$ CNT was considered. The atomic structure was relaxed using the SIESTA code [47] down to forces less than $10^{-3} \mathrm{eV} / \AA$. The junction itself is constituted of 4 periods of the CNT (around $1.7 \mathrm{~nm}$ length). The I-V curve has been obtained on the basis of a a Landauer-Büttiker Formalism :

$$
I(V)=\frac{2 e}{h} \int T(E)\left[f_{\mu_{L}}(E)-f_{\mu_{R}}(E)\right] d E,
$$

where $f_{\mu}(E)$ is the Fermi distribution and $\mu_{L}\left(\mu_{R}\right)$ the Fermi level of the left (right) electrode. We have used a non equilibrium formalism to compute the transmission for non zero bias [50]. 


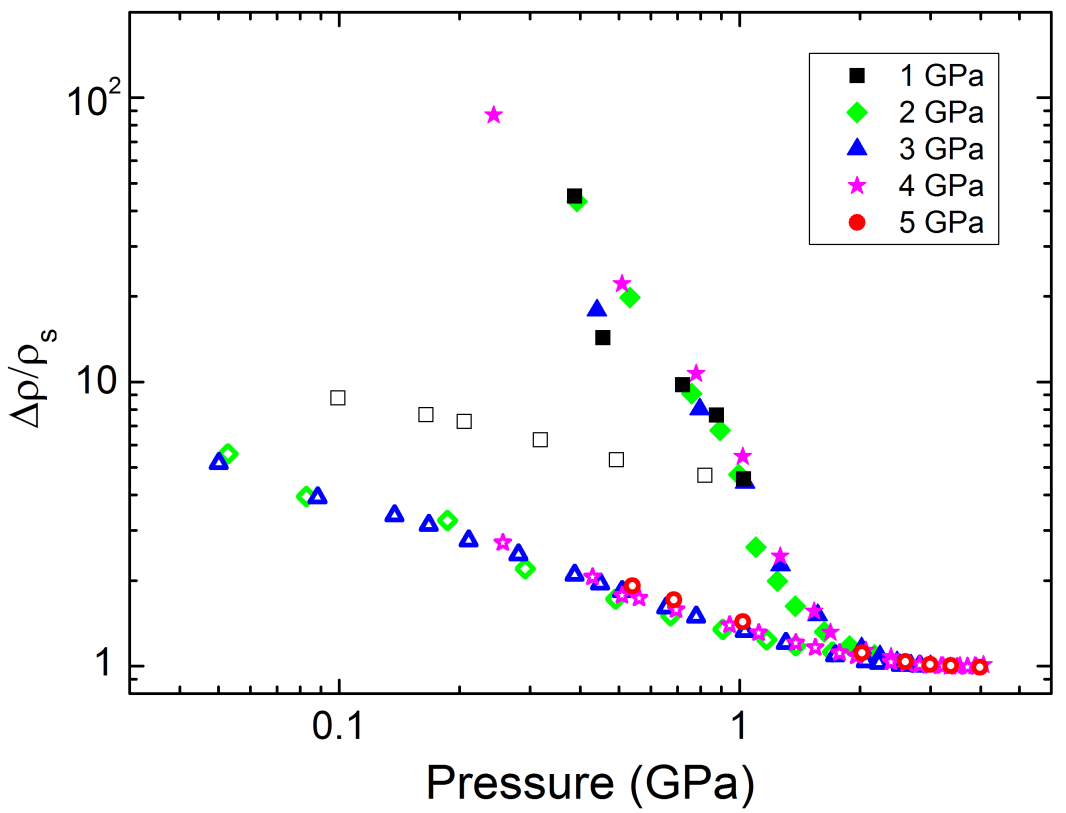

Figure 3: Normalized resistivity variation of the polyamide(PA12)-MWCNT(20\%) composite as a function of pressure in log-log scale. Filled symbols hold for compression and hollowed symbols for decompression. The symbols correspond to 5 different experiments up to a maximum pressure of $1,2,3,4$ or $5 \mathrm{GPa}$ respectively. Only the experiment with a maximum pressure of $1 \mathrm{GPa}$ showed a different behaviour under decompression.

\section{Results}

\subsection{Electrical resistivity under high pressure}

The electrical resistivity of the initial sample was measured outside the high pressure set-up giving an average value of $\sim 19 \Omega \mathrm{cm}$. The samples were then compressed up to a maximum pressure of $5 \mathrm{GPa}$. In our high pressure set-up we can measure the variation of the resistivity with pressure only after some initial compression. In fact, some compaction is needed to allow for the electrical conduction between the different parts of the set-up. The variation of the electrical resistivity of the samples was then measured in situ both during compression and decompression. Fig. 3 shows in a log-log plot the obtained results for 5 different runs with maximum pressures of 1 , 2, 3, 4 and $5 \mathrm{GPa}$, respectively. For all cases, pressure application leads to 
a resistivity reduction which we have compared with the one of a reference graphite sample studied in the same geometry. This comparison shows that the consolidation of the electrical contacts was limited to pressures below $0.2 \mathrm{GPa}$ (see the supplementary information (SI) for more details). The value of the resistivity of the composites in the set-up did not allow us to obtain any measurement at such low pressures (below $0.2 \mathrm{GPa}$ ), and hence all the obtained data can be considered as unaffected by electrode effects (see SI, Fig S3). We can thus conclude that the measured variation of the electrical resistivity with pressure is intrinsic to the composite under study. As shown in Fig. 3, the electrical resistivity decreases with pressure until saturation at a pressure $P_{s}$ of about $2 \mathrm{GPa}$. A reduction of resistivity with pressure is not an obvious effect. In fact, the opposite behavior was observed in the case of methyvinyl silicone rubber matrices containing MWCNT[51], and it was attributed to the effect of polymer chain movement on the conductive network structure of the composite, leading to the breaking of some existing conductive channels. The nature of the composite matrix plays therefore a determinant role on the pressure evolution of the nanotube network.

In the case of our highly loaded polyamide composite, the resistivity reduction during compression is characterized by a distinct change of pressure evolution at $\sim 1 \mathrm{GPa}$ and a saturation at $\sim 2 \mathrm{GPa}$. The evolution towards the saturation value is smooth and we can then define 2 regimes of behavior (below and above $1 \mathrm{GPa}$ ). Above $1 \mathrm{GPa}$ the resistivity variation with pressure becomes suddenly faster and is followed by a progressive reduction of the variation of resistivity. At about $2 \mathrm{GPa}$ the resistivity variation is no longer appreciable and the saturation regime is attained.

The resistivity discontinuity observed at about $1 \mathrm{GPa}$ is in good agreement with the recent prediction of a radial collapse pressure $P_{c}$, which depends on the nanotube internal diameter $D_{i}$ according to the law $P_{c} \propto$ $1 / D_{i}^{3}[52]$. A phase transition at a collapse pressure of $1 \mathrm{GPa}$ would then be associated to an internal diameter $D_{i}$ of about $2.5 \mathrm{~nm}[21]$. In our samples the internal diameter distribution is estimated to lie between 2 and 5.3 $\mathrm{nm}$. This distribution would give extreme collapse pressures in the range between 0.13 and $1.7 \mathrm{GPa}$. The resistivity discontinuity observed at about $1 \mathrm{GPa}$ could then be assigned in predominance to tubes with internal diameters distribution close to $2.5 \mathrm{~nm}$. This value will be later discussed in the light of our small angle neutron scattering (SANS) experiments.

How can the CNT radial collapse affect the composite conductivity? MWCNT have essentially metallic character and, as it was discussed in the 
introduction, it is not obvious if the tube collapse by itself can improve the intrinsic conductivity of the MWCNT, as both semiconductor to metal and metal to semiconductor transitions can take place in the individual tubes. We could then expect that on average the collapsed MWCNT would just preserve its metallic character. On the other hand, the tube-tube contact can be improved by the pressure effect in three different ways: (i) the evolution of the potential barrier width due to the reduction of the van der Walls tube-tube distance with pressure; (ii) the reduction of the potential barrier due to the electronic structure modification of the outer tubes and (iii) the surface enhancement of the contact area associated to a tube radial deformation. The potential barrier reduction under high pressure due to the distance decrease has been analysed by Caillier et al. [53] in nanotube-gold contacts in a pressure domain up to $1 \mathrm{GPa}$. Using this model, the expected variation of contact resistance is of $20 \%$ per GPa for tube-tube contacts. This is at least 5 times less than the resistivity variation that we observe for a sample compressed up to about $2 \mathrm{GPa}$. The pressure effect of the potential barrier reduction between tubes, due to their progressive approach, is clearly not enough to explain our measurements. In fact, an important contribution of the radial deformation would be necessary to understand our observations. We will further discuss these aspects later in the article in the context of our modeling results.

Upon pressure release we observe in Fig. 3 (open symbols) that the apparent resistivity increases, but at a much lower rate than during compression. For sample compressed with maximum pressures between 2 and $5 \mathrm{GPa}$, i.e., beyond the saturation pressure $\left(\mathrm{P}_{s}\right)$, we observe that they follow exactly the same decompression curve. The rate of resistivity variation on decompression is close to the $20 \%$ variation per GPa expected from the evolution of the pressure-induced intertube distance. This is more particularly the case of the sample that is compressed to a maximum pressure of $1 \mathrm{GPa}$. For all other samples brought beyond the $1 \mathrm{GPa}$ transition, the slope is greater, indicating that the change of behaviour observed at $1 \mathrm{GPa}$ involves a mechanism which will be determinant to understand the observed differences between the two sets of decompression curve. In spite of this, we note that the two different sets of decompression curves converge to similar values of resistivity at ambient pressure (see SI, Fig S5). This observation would be in favour of a total reversibility of the mechanisms involved in the transition observed at $1 \mathrm{GPa}$.

Surprisingly, once the samples were recovered after the pressure cycle, the resistivity measurements at ambient conditions gave values of the compos- 
ite resistivity larger than the initial ones, before the pressure cycle. Various hypothesis were examined to explain this behavior, which will be later discussed. At least two of the proposed mechanisms involve modifications to the polyamide matrix under compression. In fact, a phase transition at $3.5 \mathrm{GPa}$ has been found in polyamide fibers, related to the onset of a "contact" mechanism between neighbouring polyamide chains, i.e. the onset of possible fatigue[54]. This transition was found to be reversible, but the presence of carbon nanotubes in the polyamide matrix could potentially frustrate the reversibility or even reduce the transition pressure values due to nucleation of the polymer high-pressure phase in the tube neighbourhood. Such locally induced nucleation has been observed in the case of CNT in a Nylon matrix [55].

\subsection{High Resolution Transmission Electron Microscopy}

The high-resolution (HRTEM) observations were performed under the same conditions for 3 samples: (a) before any pressure treatment, (b) after pressure cycling up to $1 \mathrm{GPa}$ and (c) after pressure cycling up to $5 \mathrm{GPa}$. In the three cases the CNT are embedded in polyamide and under these circumstances, observations are complicated. However, CNT cross-sections can easily be observed, as shown in Fig. 4. For the pristine sample (Fig. 4 a) most of the observations showed tubes with circular cross-sections. We observed nevertheless a small number of deformed tubes. The contrast of the images is not enough to allow for a precise determination of the number of tubes walls. The radially deformed tubes in Fig 4 a.2 and Fig4 a.3 are identified, respectively, as tubes of 7-9 walls and a diameter of $\sim 6.5 \mathrm{~nm}$ and tubes of 6-7 walls and a diameter of $\sim 7 \mathrm{~nm}$. This means that these deformed tubes correspond to points just below the shadowed zone in Fig. 1, consistently with the fact that they are just ovalized and not collapsed. The situation is reversed for sample the (b) in Fig. 4b: after cycling up to $1 \mathrm{GPa}$, the observed tubes appeared to be in majority deformed and it is more difficult to find tubes with circular cross-sections. The images of Fig. 4b.2 and Fig. 4b.3 are particularly interesting, as they show in a same tube two sections having different deformation of their cross-sections. In particular in Fig. 4 b.3 we observe that the cross-section deformation changes remarkably after the contact with another tube. The images in Fig. 4c refer to the cross-section of tubes in the sample which was brought to $5 \mathrm{GPa}$. We can appreciate that in this sample, an important number of tubes showed stronger deformations than in the two previous samples cases. These micrographs represent clear evidence of the 


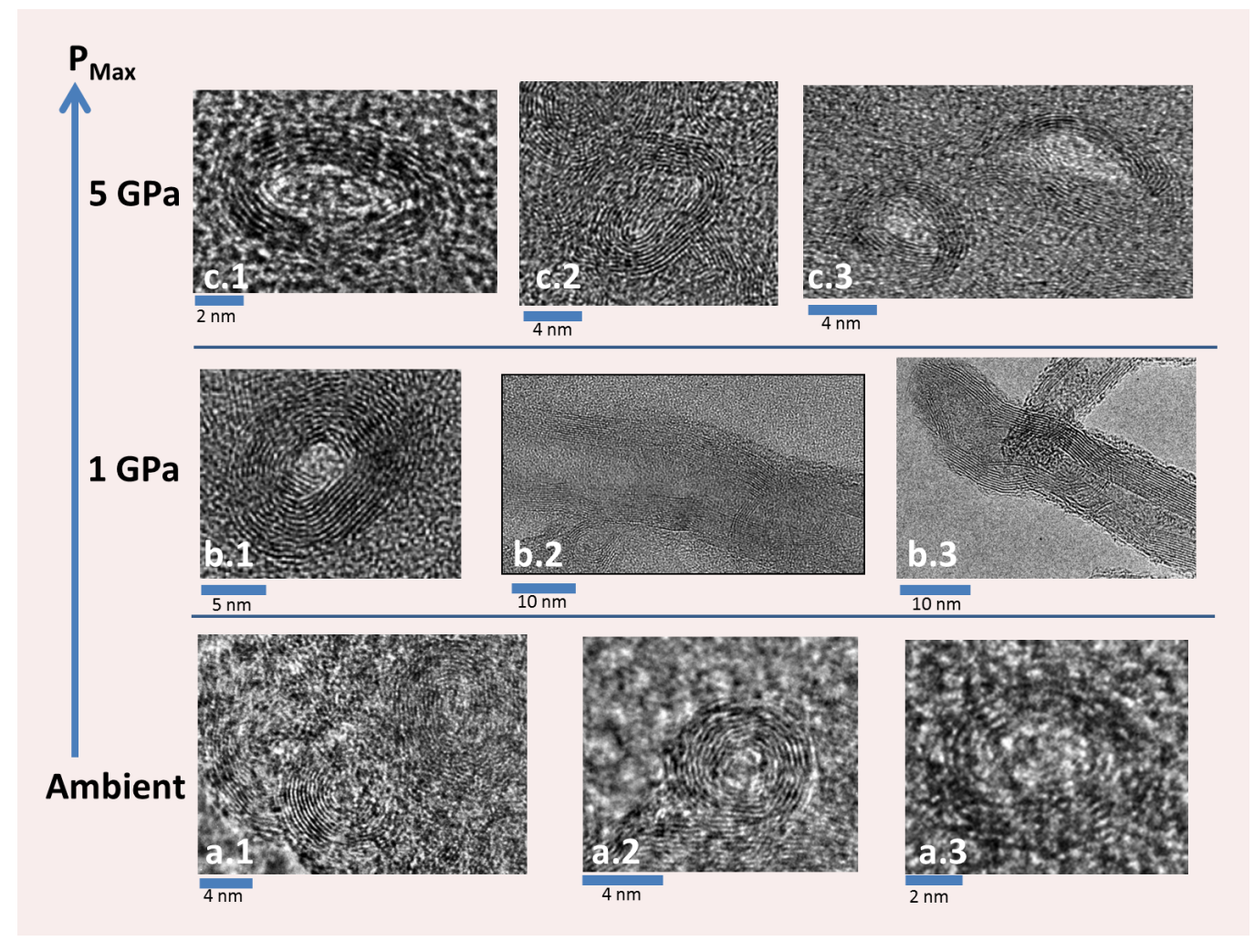

Figure 4: HRTEM micrographs of the composite at ambient conditions. (a1 to a3) before the pressure cycle. (b1 to b3) after a pressure cycle up to $1 \mathrm{GPa}$.(c1 to c3) after a pressure cycle up to $5 \mathrm{GPa}$. 
CNT transformation, from a circular cross-section to elliptical/oval-shape due to the pressure effect. It is worth noting that we could also observe that some nanotubes did not undertake any modification after the pressure cycle. These nanotubes corresponded to those having small internal diameters which then provide a better mechanical support as it has been observed in DWCNT $[56,57]$. The obtained HRTEM images are the first direct evidence of pressure induced cross-section modification in CNT.

\subsection{Small Angle Neutron Scattering}

As HRTEM information is of local nature, it is interesting to complete the structural information with a technique that gives an average image of the sample structure. This is the case of small angle neutron scattering (SANS). This technique provides information on the mesoporous structure of materials, which is complementary to HRTEM. SANS spectra were taken on samples at ambient conditions before and after a pressure cycle up to $5 \mathrm{GPa}$. Fig. 5 shows the scattering intensity for the pristine sample before and after a pressure cycle up to $5 \mathrm{GPa}$. The neutron scattering vector is perpendicular to the compression direction. The scattering signal is due to the contrast between the matrix and the MWCNT. To fit it, we model nanotubes as objects having characteristic dimensions at two different scales. In the low scattering vector $q$ range we consider the contribution of a chain of coherent scatters (Kuhn segments) characterized by their length, the Kuhn length $(K)$. In the high- $q$ the scattering signal is modeled by a tube of internal diameter $\left(D_{i}\right)$ having thickness $(t)$.

At ambient pressure, the initial sample gives a good fit with $D_{i}=3.7 \pm 0.3$ $\mathrm{nm}, t=2.6 \pm 0.2 \mathrm{~nm}$ and $K=42 \pm 3 \mathrm{~nm}$, which corresponds to an average tube of external diameter $D_{e}=8.9 \pm 1.4 \mathrm{~nm}$ having approximately 8 walls. This is in quite good agreement with the nominal values given by the nanotube manufacturer (average external diameter of $12 \mathrm{~nm}$ and 10-15 walls). Differences can be attributed to the simplicity of the model, which does not account for the distribution of tube diameters. The obtained value for the average internal diameter $D_{i}=3.7$ would give a collapse pressure of $0.35 \mathrm{GPa}$, which differs from the $1 \mathrm{GPa}$ value at which we find the discontinuity in the resistivity behaviour. Nevertheless the comparison should be done with caution, as different factors can modify both the internal diameter and the collapse pressure. As already mentioned the SANS model does not include a geometry distribution of the CNT and the obtained $D_{i}$ must be treated with caution. On the other hand the calculations yield a large dispersion of 


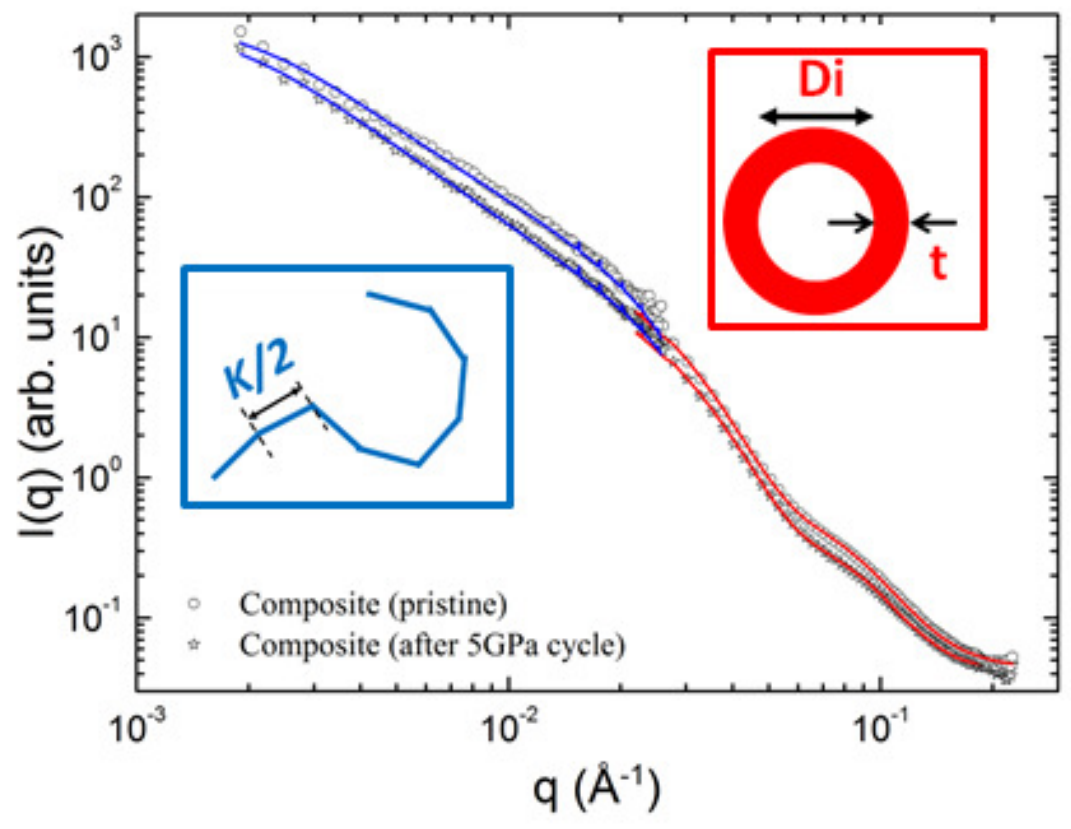

Figure 5: Small angle neutron scattering intensity versus scattering vector $q$ for the MWCNT(20\%)@PA12 sample at ambient pressure and after a pressure cycle up to 5 GPa. Continuous lines correspond to the best fits to two models fitted in two different $q$-ranges. For low $q$-values the nanotubes where modelized as a concatenation of Kuhn segments of length $K$ (see text for details). In the higher- $q$ values the scattered intensity was modelized as originated by cylinders of internal diameter $D_{i}$ and thickness $t$. 
values for the collapse pressure, pointing to the non-negligible error bar of the predictions[21]. These predictions were also obtained for nanotube bundles. In addition, as commented, the interaction with the polyamide matrix can also contribute to a modification of the collapse pressure. Altogether, the agreement between calculations and experiments needs to be evaluated considering all these sources of incertitude.

After the pressure cycle up to $5 \mathrm{GPa}$, the obtained values are $D_{i}=3.5 \pm 0.3$ $\mathrm{nm}, t=3.1 \pm 0.2 \mathrm{~nm}$ and $K=31 \pm 4 \mathrm{~nm}$, which means a reduction of the Kuhn length and an increment in the projection of the tube thickness in the direction perpendicular to the applied pressure. This increasing of $t$ of $\sim$ $20 \%$ is then in rather good agreement with the HRTEM observations. The SANS signal provides an average of the contribution of all tubes - deformed or not - and this could explain that the fit with such a simplified model does not produce a significant modification of $D_{i}$ after the pressure cycle.

\subsection{Numerical Modeling}

We have explored numerically the effect of the tube collapse on a system of two SWCNT. The system under consideration is composed of two $(18,0)$ carbon nanotubes in close contact as depicted in Fig. 6.a. We have considered both the case of the contact between two pristine nanotubes and the contact between two fully collapsed nanotubes. The collapsed carbon nanotube structure has been obtained by atomic relaxation under pressure using density-functional tight-binding[21]. The computed collapse pressure is $2.8 \mathrm{GPa}$.

In order to check the effect of the collapse on the system's electrical resistance, we investigated its variation with the distance between the two nanotubes. This distance can be fixed using the inter-layer distance variation with pressure experimentally measured in graphite[58]. Calculations are performed using the Landauer-Büttiker formalism based on density functional theory electronic states.

In our model the relative position of the tubes at the junction in the collapsed state has been described in two different configurations. The first one corresponds to a situation with the highest symmetry, i.e, the plane defined by the two tubes axis in the collapsed form is a plane of symmetry of the system (Fig. 6.b), as in the non-collapsed geometry. The pressure induced reduction of the inter-tube distance in the collapsed form is then governed by the end-lobes of the tubes. TEM images in collapsed DWNT bundles show a tube packing structure leading to a relative position of the 


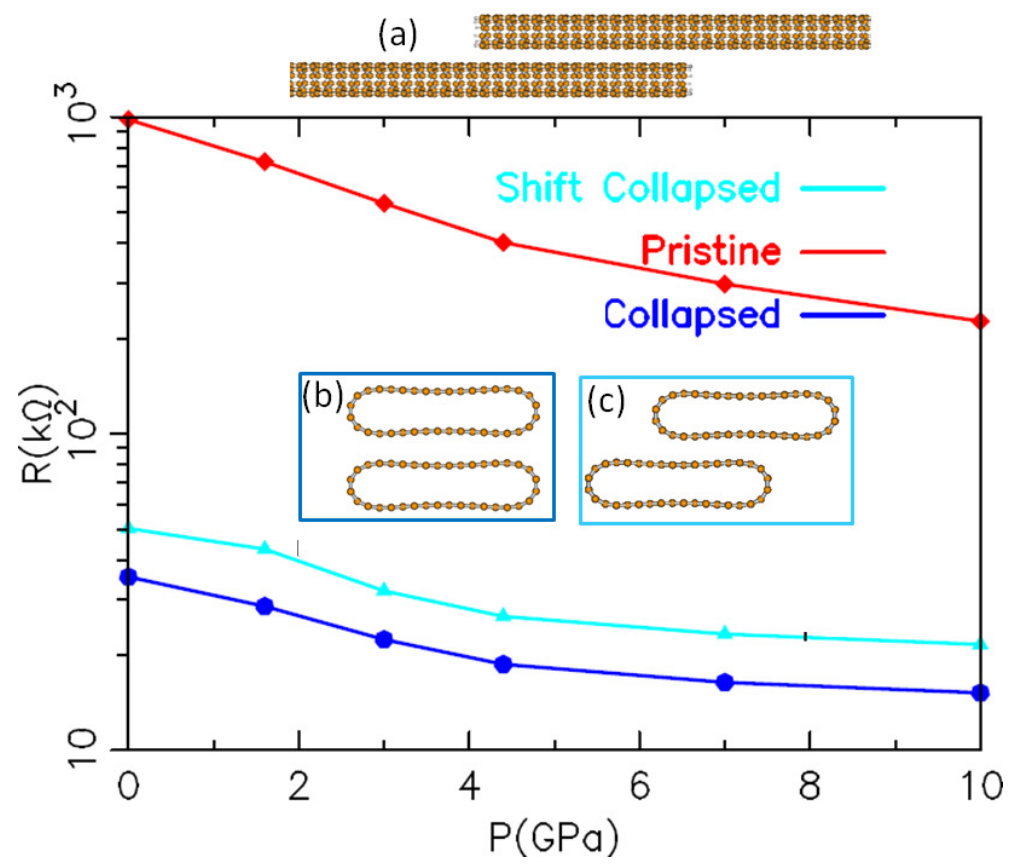

Figure 6: Modelling of resistance evolution with pressure of two $(18,0)$ SWCNT nanotube contacted along the axis as shown in (a). Three different cases are considered: the two tubes cross-section remain circular during the full compression, i.e., in their pristine state(red squares); the two tubes are in the collapsed phase and not shifted laterally (b)(blue circles); and the two tubes are collapsed in the side-shifted geometry)(c) (magenta triangles). In all cases only the distance between tubes is varied with pressure and the tube geometry is kept constant.(Color online) 
collapsed tubes in which the tubes slide laterally reducing in that way the intertube distance in the flattened part[14]. In high diameter tubes, this shifted-junction geometry will lead to larger regions of the tube at the van der Waals distance at a given pressure in the collapsed geometry. We have then also considered such a geometry as shown in Fig. 6.c.

We observe that for the three cases investigated in this work (pristine, symmetrically collapsed and shifted-junction), between ambient pressure and $4 \mathrm{GPa}$ the resistance reduction ranges between 12 and $14 \% \mathrm{GPa}^{-1}$. This value is smaller than the $20 \% \mathrm{GPa}^{-1}$ which was derived experimentally in Ref.[53]. The symmetrically contacted collapsed $(18,0)$ SWCNT have a resistance 30 to 15 times smaller than the resistance of the contacted pristine tubes. A phase change between circular to collapsed (or highly radially deformed) geometry will decrease the electrical resistance. In the case of shifted-collapsed tubes, the pressure behaviour of their resistance is analogous to the symmetriccollapsed tubes but with a resistance about 1.5 times larger. The observed differences between the pristine and collapsed geometries is mainly coming from the fact that the pristine tubes have a small gap (about $20 \mathrm{meV}$ ), while we find that the collapsed tubes have a zero gap. However, we have found that for $(12,0)$ nanotubes (which have similar gap for pristine and collapsed structure) the ratio between the resistance of the collapsed structure and the pristine one is still of the order of 5 . This gain is strongly dependent on the geometrical details of the contact and on the nanotube size.

In order to explore the importance of the presence of junctions of collapsed/noncollapsed regions in a tube as it is observed in the HRTEM images (Fig. 4 b.2 and b.3), we have studied a system corresponding to a junction between a pristine $(12,0)$ carbon nanotube $(\mathrm{CNT})$ and a fully collapsed section of the same tube as depicted in Fig. 7.a. and .b In our approach the collapsed part of the tube corresponds to the pressure relaxed structure. This approach differs from other studies in which uniaxial stress has been used to modify the tube radial cross-section $[59,35]$.

The aim is to study the influence of the electronic backscattering on the electronic transport. Fig. 7.c corresponds to the zero bias transmission of this junction (for computational details see [48]). We have represented both the transmission of the pristine CNT, the collapsed CNT and the transmission through the junction.

The tiny gap of the $(12,0) \mathrm{CNT}$ is almost unchanged with pressure (for $(\mathrm{n}, \mathrm{n}) \mathrm{CNT}$, a metal to semiconductor transition is observed). The main influence of the collapse on the transmission is the occurrence of $2 \sigma$ type bands 
(a)
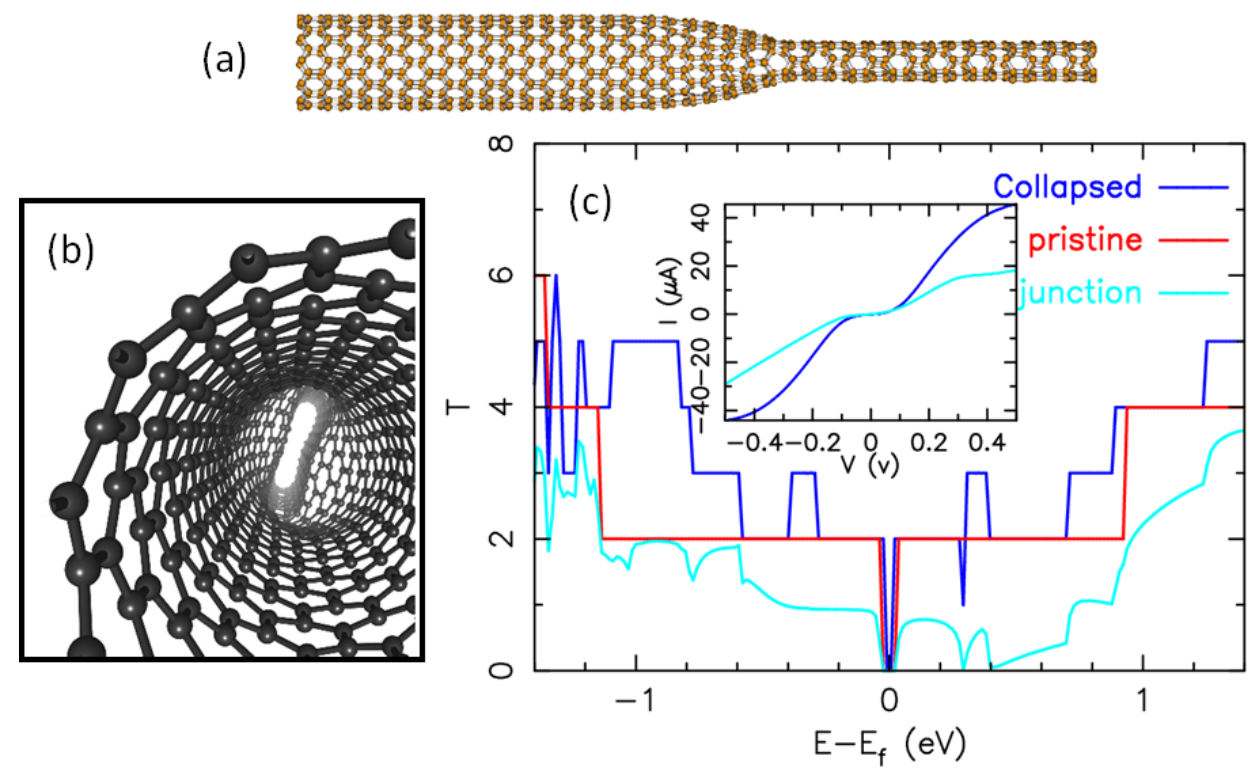

Figure 7: ( $\mathrm{a}$ and b) Model of a junction between a non-collapsed and a collapsed region in a $(12,0)$ SWCNT: (a) lateral view; (b) perspective view from inside the tube. (c) corresponding zero biais tansmission of the junction as compared with the fully collapsed and the fully pristine tube. The inset shows the calculated I-V curve of the junction compared to the one of the collapsed tube. 
close to the Fermi level, which leads to a larger transmission for the collapsed tube compared with the pristine one. We observe a large backscattering on the transmission of the junction i.e. the transmission for the $\pi$ and $\pi^{*}$ bands is lower than the pristine and collapsed transmission. This backscattering is not symmetric for the conduction and valence band. For the valence band, the transmission is reduced by half. For the conduction band, it almost goes to zero.

The calculated I-V curve shown in the inset of Fig. 7.c provides evidence that the observed backscattering on the transmission is leading to a lower current for the junction compared with the current of the collapsed CNT, i.e., the junction limits the transmission with respect to the collapsed tube. The presence of such junctions will then lead to a conductivity reduction of the composite. We have verified that the obtained results remain unchanged when other junction lengths are considered.

Our modeling findings on contacted SWCNT in two different states (pristine and fully collapsed) constitute a first step to the understanding of how the pressure modification of the radial cross-section of CNT affects the electrical performances of the tube percolated network. Different extensions of these calculations would be needed as: i) the modeling of multiple wall nanotubes; ii) the study of the different states of radial deformation. Another important factor is the difference of diameter between our modeled tubes and the MWCNT system studied. For large diameter MWCNT, considering our HRTEM images, oval or even race-track type cross-sections are structures easier to obtain. In such a case we may expect a much important reduction of the percolated tube resistivity with pressure due to large tube-tube contact surface changes on radial deformation. Another important aspect is that MWCNT will be composed of different chiralities and the intrinsic conduction will be dominated by those tubes having the highest conductance after collapse. In MWCNT we can then have a high probability of an intrinsic metallic character (equivalent or may be improved with respect to the SWCNT in which we can guess that some percolation paths will be switch on and others off. Those considerations lead to expect an even more important resistivity reduction with pressure in MWCNT networks than for SWCNT.

\section{Discussion}

From the different experimental results and theoretical modeling we can now propose a coherent scenario for the full pressure cycle of the composite. 
The MWCNT geometry distribution is quite large and already at ambient pressure some tubes do not present a perfectly circular cross-section. Pressure application progressively reduces the intertube distance and continues to deform those tubes having the larger internal diameters and the smallest number of walls. As a consequence the electrical resistivity of the composite decreases both (i) because of the intertube barrier reduction due to tube-tube distance decrease and (ii) due to the tube cross-section deformation which allows for an intrinsic tube resistance reduction and a better junction transmission. At about $1 \mathrm{GPa}$, the collapse process becomes suddenly more important, in connection with the increase of contribution (ii) to the resistivity reduction. Collapse at this particular pressure can have different origins: (a) the predominance of MWCNT having those geometrical conditions allowing for their radial collapse at that pressure; (b) a contribution from a matrix volume reduction which could drive the MWCNT transition; (c) the effect of the crystallization of some water present in the polyamide (the transition from liquid water to ice VII takes place at about $1 \mathrm{GPa}$ at ambient temperature). Both (b) or (c) would lead to a sudden appearance of an inhomogeneous stress field which would facilitate the collapse of MWCNT. Independently of the nature of the factors participating in the massive collapse of MWCNT, at $1 \mathrm{GPa}$ the consequent improvement of the electric contact between tubes or even intrinsic modifications of the deformed-tube resistivity leads to a marked resistivity reduction, which progresses up to the complete collapse of a large fraction of tubes at about $2 \mathrm{GPa}$. From this pressure, further pressure application up to $5 \mathrm{GPa}$, does not significantly change the composite resistivity.

Upon pressure release we can distinguish two cases: depending if the pressure is released before the collapse pressure of about $1 \mathrm{GPa}$ is reached, or after $2 \mathrm{GPa}$, when the collapse process has been essentially completed. The intermediate regime has not been explored. In both cases the resistivity increases upon pressure release, and it is brought to approximately the same values at ambient pressure independently of the maximum attained pressure. The HRTEM images showed differences in the deformation rates of tubes brought back from a maximum pressure of $1 \mathrm{GPa}$ and $5 \mathrm{GPa}$. They also show that in the inter-tubes contact zone of tubes having been compressed up to $1 \mathrm{GPa}$, there is a stronger tube deformation. We can then argue that even if the tubes cross-sections can be more or less deformed depending on the maximum compression pressure, similar deformations can be obtained at the contacts, affecting the electronic transmission of the recovered samples. The 
observed increase in the number of deformed tubes after a pressure cycle up to $1 \mathrm{GPa}$ is related to the irreversible (or partially irreversible) radial deformation of those tubes which have geometrical parameters that allow for their deformation in the low pressure regime (below $1 \mathrm{GPa}$ ). In fact, for these tubes the elastic contribution which would allow them to recover their initial shape is less significant (they have larger internal diameter) and can be counterbalanced by both matrix interactions and inter-nanotube interactions between the flattened walls. For tubes brought up to $5 \mathrm{GPa}$ the HRTEM images show stronger irreversible changes, with tubes showing radial crosssections with profiles which could even be associated in some cases to higher order corrugation modes[60], i.e., having more than two high curvature zones in their contour (see (Fig. 4c.2). Nevertheless the measured resistivity at decompression seemed to converge to similar values to the ones observed for the sample compressed to $1 \mathrm{GPa}$. This could be due to the presence of other compensating mechanisms appearing at higher deformation, but the present results do not permit further insights on this aspect.

Our calculations support the idea that the conductivity should be improved after a full pressure cycle due both to the improved intrinsic conductivity of the collapsed tubes and the improvement of the transmission on the collapsed tube-tube junctions. It is difficult to evaluate the weight of each contribution in the measured composite as our simulations concerned only SWCNT of a restricted diameter.

In the 5 experiments performed, the resistivity of the composite upon pressure release reaches values orders of magnitude lower than the initial one. However, the resistivity suddenly increases after the last pressure point in the decompression curve of Fig. 3 (not shown). The pressure at which this sudden increase was observed lies in the interval between 0.05 and $0.5 \mathrm{GPa}$. Resistivity measurements of the recovered samples show unexpectedly very high resistivity values, about one order of magnitude higher than the initial one. This effect can be due to the electrical disruption between the sample and the metallic contacts (this was observed at $0.5 \mathrm{GPa}$ in experiments on graphite). This implies that in the last few kbars $(1 \mathrm{kbar}=0.1 \mathrm{GPa})$ of the decompression the electrical percolation network suffered some type of disruption. Different reasons can be at the origin of this observation : i) cracks in the matrix; ii) a massive appearance of collapsed/non-collapsed junctions; iii) a matrix volume increase during the decompression, specially in the last kbars. In fact, if the tube-matrix adhesion forces are strong enough, this will lead to axial pulling forces in the tubes which will eventually break 
the tubes and disrupt the network conductivity.

Optical and SEM images of the recovered samples did not show any signs of cracks in the polymer matrix and we can then discard this hypothesis. It is difficult to decide from the two other hypotheses. Concerning explanation ii), Chang et al. [61] have shown by MD simulations that the collapse in SWCNT propagates very rapidly along the tube driven by the van der Waals potential energy but only for tube diameters larger than $3.5 \mathrm{~nm}$. The minimum internal diameter for collapse propagation should decrease with pressure application and increase with a increasing number of tube walls or due to tube-matrix interactions, making not obvious if such mechanism would apply in our case. Whatever the case, on pressure release, some of our HRTEM images show the existence of collapsed/non-collapsed junctions. Our calculations show that they can limit the conductivity of the final composite. Concerning the third possible explanation, iii), it has been observed that under high pressure the extrusion forces caused by the matrix can break carbon nanotubes[22] and in fact our SANS measurements showed a reduction of the Kuhn length which can be related with such an effect. The background signal evolution of the Raman spectra (see SI Fig. S6) does not allow us to decide on a clear modification of the D-band, which would have been a signature of the creation of tube defects by a certain mechanisms. In all cases, a heat treatment during decompression could be explored in order to avoid the observed percolation disruption, either by relaxing the matrix-tube adhesion and avoiding tube breaking or by relaxing the mechanism which stops the propagation of collapsed or non-collapsed regions. Finally we should mention that the compression cycling does not show any sign of a favourable release mechanism of carbon nanotubes from the matrix. Compressive stress then, as other mechanical stresses[62] does not favour nanoparticle release in carbon nanotube-polyamide composites.

\section{Conclusions}

We have shown that the conductivity of commercially available MWCNT polyamide composites can be enhanced by a pressure treatment up to about $1 \mathrm{GPa}$ thanks to the improvement of tube-tube contacts through radial deformation of the tube and/or the intrinsic changes in the tube resistivity due to radial deformation. A further increase of pressure beyond $1 \mathrm{GPa}$ does not improve the final resistivity on decompression. This work points to the possibility of adjusting the pressure treatment for pressures below $1 \mathrm{GPa}$ in 
order to optimize the electrical percolation network for low loaded composites. The use of MWCNT with larger internal diameters or a smaller number of walls would allow to further reduce the pressure needed for the composite optimization. Finally, we have given for the first time direct evidence of the pressure induced radial deformation of CNT through HRTEM images supported by SANS experiments.

\section{Acknowledgements}

We acknowledge financial support by the ANR program P2N under Contract ANR-11-NANO-025 "TRI-CO". We are indebted to the Arkema company for providing samples and associated information. We acknowledge technical support of F. Legrand (ILM, Lyon). The transmission electron microscopy studies were conducted at the Laboratorio de Microscopias Avanzadas (LMA) at the Instituto de Nanociencia de Aragon (INA) - Universidad de Zaragoza (Spain). R.A. acknowledges funding from the Spanish Ministerio de Economia y Competitividad (FIS2013-46159-C3-3-P). The research leading to these TEM results has received funding from the from the European Union Seventh Framework Program under Grant Agreement 604391 Graphene Flagship, as well as from EU H2020 ETN project "Enabling Excellence" Grant Agreement 642742.

\section{Suppementary information}

Supplementary experimental procedures and characterizations are included.

\section{References}

[1] Coleman, J.N., Khan, U., Blau, W.J., Gunko, Y.K.. Small but strong: A review of the mechanical properties of carbon nanotube-polymer composites. Carbon 2006;44:1624 - 1652 .

[2] Spitalsky, Z., Tasis, D., Papagelis, K., Galiotis, C.. Carbon nanotubepolymer composites: Chemistry, processing, mechanical and electrical properties. Progress in Polymer Science 2010;35(3):357 - 401.

[3] Arash, B., Wang, Q., Varadan, V.. Mechanical properties of carbon nanotube/polymer composites. Scientific Reports 2014;4:6479. 
[4] Zeng, Y., Ci, L., Carey, B.J., Vajtai, R., Ajayan, P.M.. Design and reinforcement: Vertically aligned carbon nanotube-based sandwich composites. ACS Nano 2010;4(11):6798-6804.

[5] Bauhofer, W., Kovacs, J.Z.. A review and analysis of electrical percolation in carbon nanotube polymer composites. Composites Science and Technology 2009;69(10):1486 - 1498.

[6] Chopra, N.G., Benedict, L.X., Crespi, V.H., Cohen, M.L., Louie, S.G., Zettl, A.. Fully collapsed carbon nanotubes. Nature 1995;377:135138.

[7] Yu, M.F., Dyer, M.J., Ruoff, R.S.. Structure and mechanical flexibility of carbon nanotube ribbons: An atomic-force microscopy study. Journal of Applied Physics 2001;89(8):4554-4557.

[8] Motta, M., Moisala, A., Kinloch, I., Windle, A.. High performance fibres from "dog bone" carbon nanotubes. Advanced Materials 2007;19:3721-3726.

[9] Benedict, L.X., Chopra, N.G., Cohen, M.L., Zettl, A., Louie, S.G., Crespi, V.H.. Microscopic determination of the interlayer binding energy in graphite. Chemical Physics Letters 1998;286:490 - 496.

[10] Xiao, J., Liu, B., Huang, Y., Zuo, J., Hwang, K.C., Yu, M.F.. Collapse and stability of singleand multi-wall carbon nanotubes. Nanotechnology 2007;18(39):395703.

[11] Shima, H.. Buckling of carbon nanotubes: A state of the art review. Materials 2011;5(1):47.

[12] Chopra, N., Ross, F., Zettl, A.. Collapsing carbon nanotubes with an electron beam. Chemical Physics Letters 1996;256(3):241-245.

[13] Gong, T., Zhang, Y., Liu, W., Wei, J., Jia, Y., Wang, K., et al. Structural transformation of double-walled carbon nanotube bundles into multi-walled carbon nanotubes induced by current treatment. Diamond and Related Materials 2008;17(2):158 - 161.

[14] Zhong, X.H., Wang, R., Liu, L.B., Kang, M., Wen, Y.Y., Hou, F., et al. Structures and characterizations of bundles of collapsed doublewalled carbon nanotubes. Nanotechnology 2012;23(50):505712. 
[15] Choi, D.H., Wang, Q., Azuma, Y., Majima, Y., Warner, J.H., Miyata, Y., et al. Fabrication and Characterization of Fully Flattened Carbon Nanotubes: A New Graphene Nanoribbon Analogue. Sci Rep 2013;3:1617.

[16] Tangney, P., Capaz, R.B., Spataru, C.D., Cohen, M.L., Louie, S.G.. Structural transformations of carbon nanotubes under hydrostatic pressure. Nano Letters 2005;5(11):2268-2273.

[17] Blancon, J.C., Ayari, A., Marty, L., Bendiab, N., San-Miguel, A.. Electronic Transport in Individual Carbon Nanotube Bundles under Pressure. Journal of Applied Physics 2013;114:143704.

[18] Zhang, S., Khare, R., Belytschko, T., Hsia, K.J., Mielke, S.L., Schatz, G.C.. Transition states and minimum energy pathways for the collapse of carbon nanotubes. Phys Rev B 2006;73:075423.

[19] Elliott, J.A., Sandler, J.K.W., Windle, A.H., Young, R.J., Shaffer, M.S.P.. Collapse of single-wall carbon nanotubes is diameter dependent. Physical Review Letters 2004;92:095501.

[20] Hasegawa, M., Nishidate, K.. Radial deformation and stability of single-wall carbon nanotubes under hydrostatic pressure. Phys Rev B 2006;74:115401.

[21] Cerqueira, T.F.T., Botti, S., San-Miguel, A., Marques, M.A.L.. Density-functional tight-binding study of the collapse of carbon nanotubes under hydrostatic pressure. Carbon 2014;69:355-360.

[22] Caillier, C., Machon, D., San-Miguel, A., Arenal, R., Montagnac, G., Cardon, H., et al. Probing high-pressure properties of singlewall carbon nanotubes through fullerene encapsulation. Phys Rev B 2008;77(12):125418.

[23] Yao, M., Wang, Z., Liu, B., Zou, Y., Yu, S., Lin, W., et al. Raman signature to identify the structural transition of single-wall carbon nanotubes under high pressure. Phys Rev B 2008;78(20):205411.

[24] Torres-Dias, A.C., Cambr, S., Wenseleers, W., Machon, D., SanMiguel, A.. Chirality-dependent mechanical response of empty and 
water-filled single-wall carbon nanotubes at high pressure. Carbon 2015;95:442 - 451 .

[25] Zhang, Y., Liu, G., Qiang, H., Li, G.. Investigation of doublewalled carbon nanotubes embedded in an elastic medium using the energy method. International Journal of Mechanical Sciences 2006;48(1):53 -61 .

[26] Pugno, N.M.. The design of self-collapsed super-strong nanotube bundles. Journal of the Mechanics and Physics of Solids 2010;58(9):13971410 .

[27] Pugno, N.M., Elliott, J.A.. Buckling of peapods, fullerenes and nanotubes. Physica E: Low-dimensional Systems and Nanostructures 2012;44(6):944 - 948 .

[28] Mazzoni, M.S.C., Chacham, H.. Bandgap closure of a flattened semiconductor carbon nanotube: A first-principles study. Applied Physics Letters 2000;76(12):1561-1563.

[29] Lammert, P.E., Zhang, P., Crespi, V.H.. Gapping by squashing: Metal-insulator and insulator-metal transitions in collapsed carbon nanotubes. Phys Rev Lett 2000;84:2453-2456.

[30] Charlier, A., McRae, E., Heyd, R., Charlier, M.F.. Metalsemiconductor transitions under uniaxial stress for single- and doublewalled carbon nanotubes. Journal of Physics and Chemistry of Solids 2001;62(3):439 - 444 .

[31] Gülseren, O., Yildirim, T., Ciraci, S., Kılıç, i.m.c.. Reversible bandgap engineering in carbon nanotubes by radial deformation. Phys Rev B 2002;65:155410.

[32] Gómez-Navarro, C., Sáenz, J.J., Gómez-Herrero, J.. Conductance oscillations in squashed carbon nanotubes. Phys Rev Lett 2006;96:076803.

[33] Chen, D., Sasaki, T., Tang, J., Qin, L.C.. Effects of deformation on the electronic structure of a single-walled carbon nanotube bundle. Phys Rev B 2008;77:125412. 
[34] Nishidate, K., Hasegawa, M.. Universal band gap modulation by radial deformation in semiconductor single-walled carbon nanotubes. Phys Rev B 2008;78:195403.

[35] Mouri, M., Ogawa, M., Souma, S.. Influence of geometrical deformation and electric field on transport characteristics through carbon nanotubes. Journal of Applied Physics 2012;112(11):114328.

[36] Okuno, Y., Saito, Y., Kawata, S., Verma, P.. Tip-enhanced raman investigation of extremely localized semiconductor-to-metal transition of a carbon nanotube. Phys Rev Lett 2013;111:216101.

[37] Giusca, C.E., Tison, Y., Silva, S.R.P.. Evidence for metalsemiconductor transitions in twisted and collapsed double-walled carbon nanotubes by scanning tunneling microscopy. Nano Letters 2008;8(10):3350-3356.

[38] Byrne, M.T., Gun'ko, Y.K.. Recent advances in research on carbon nanotubepolymer composites. Advanced Materials 2010;22(15):16721688. URL: http://dx.doi.org/10.1002/adma. 200901545. doi:10.1002/adma.200901545.

[39] Sandler, J., Pegel, S., Cadek, M., Gojny, F., van Es, M., Lohmar, J., et al. A comparative study of melt spun polyamide-12 fibres reinforced with carbon nanotubes and nanofibres. Polymer 2004;45(6):2001 - 2015. URL: http://www.sciencedirect.com/science/article/pii/S0032386104000473. doi:http://dx.doi.org/10.1016/j.polymer.2004.01.023.

[40] Jouni, M., Faure-Vincent, J., Fedorko, P., Djurado, D., Boiteux, G., Massardier, V.. Charge carrier transport and low electrical percolation threshold in multiwalled carbon nanotube polymer nanocomposites. Carbon 2014;76:10 - 18. URL: http://www.sciencedirect.com/science/article/pii/S0008622314003637. doi:http://dx.doi.org/10.1016/j.carbon.2014.04.031.

[41] Synth. Ph.D. thesis; ????

[42] Versavaud, S., Rgnier, G., Gouadec, G., Vincent, M.. Influence of injection molding on the electrical properties of polyamide 12 filled with 
multi-walled carbon nanotubes. Polymer 2014;55(26):6811 - 6818. URL: http://www. sciencedirect.com/science/article/pii/S0032386114009471. doi:http://dx.doi.org/10.1016/j.polymer.2014.10.038.

[43] San-Miguel, A., Toulemonde, P.. High-pressure properties of group iv clathrates. High Pressure Research 2005;25(3):159-185.

[44] Cotton, J.P.. Introduction to Scattering Experiments. Neutrons X-Rays Light Scatt. Methods. Amsterdam: Elsevier; 1991.

[45] Brûlet, A., Lairez, D., Lapp, A., Cotton, J.P.. Improvement of Data Treatment in Small-Angle Neutron Scattering. Journal of Applied Crystallography 2007;40(1):165-177.

[46] Machon, D., Toulemonde, P., McMillan, P.F., Amboage, M., Muñoz, A., Rodríguez-Hernández, P., et al. High-pressure phase transformations, pressure-induced amorphization, and polyamorphic transition of the clathrate $\mathrm{Rb}_{6.15} \mathrm{Si}_{46}$. Phys Rev B 2009;79:184101.

[47] Soler, J.M., Artacho, E., Gale, J.D., García, A., Junquera, J., Ordejón, P., et al. The siesta method for ab initio order-n materials simulation. J Phys: Condens Matter 2002;14:2745-2779.

[48] Adessi, C., Roche, S., Blase, X.. Reduced backscattering in potassiumdoped nanotubes: Ab initio and semiempirical simulations. Phys Rev B 2006;73:125414.

[49] Datta, S.. Electronic transport in mesoscopic systems. Cambridge, United Kingdom: Cambridge University Press; 1995.

[50] Brandbyge, M., Mozos, J.L., Ordejón, P., Taylor, J., Stokbro, K.. Density-functional method for nonequilibrium electron transport. Phys Rev B 2002;65:165401.

[51] Jiang, M.J., Dang, Z.M., Xu, H.P., Yao, S.H., Bai, J.. Effect of aspect ratio of multiwall carbon nanotubes on resistance-pressure sensitivity of rubber nanocomposites. Applied Physics Letters 2007;91(7):072907.

[52] Alencar, R.S., Torres-Dias, A.C., Cerqueira, T.F.T., Cui, W., Botti, S., Marques, M., et al. Pressure-induced radial collapse in few-wall carbon nanotubes: A combined theoretical and experimental study. Submited 2016; 
[53] Caillier, C., Ayari, A., Gouttenoire, V., San Miguel, A., Jourdain, V., Picher, M., et al. Gold contact to individual metallic carbon nanotubes: A sensitive nanosensor for high-pressure. Applied Physics Letters 2010;97:173111.

[54] Colomban, P., Sagon, G., Lesage, M., Ramirez, J.M.H.. Micro-raman study of the compressive behaviour of advanced pa66 polyamide fibres in a diamond-anvil cell. Vibrational Spectroscopy 2005;37(1):83 - 90.

[55] Jia, Z., Zeng, F., Yuan, Q., Misra, R.. Carbon nanotube-induced structure and phase evolution in polymer-based nanocomposites crystallized at elevated pressures. Materials Science and Engineering: B 2012;177(9):666 - 672 .

[56] Aguiar, A.L., Barros, E.B., Capaz, R.B., Souza Filho, A.G., Freire, P.T.C., Mendes Filho, J., et al. Pressure-induced collapse in doublewalled carbon nanotubes: Chemical and mechanical screening effects. J Phys Chem C 2011;115(13):5378-5384.

[57] Aguiar, A.L., Capaz, R.B., Souza Filho, A.G., San-Miguel, A.. Structural and phonon properties of bundled single- and double-wall carbon nanotubes under pressure. J Phys Chem C 2012;116(42):2263722645 .

[58] Hanfland, M., Beister, H., Syassen, K.. Graphite under pressure: Equation of state and first-order raman modes. Phys Rev B 1989;17:12598-12603.

[59] Lu, J.Q., Wu, J., Duan, W., Gu, B.L.. Effects of finite deformed length in carbon nanotubes. Applied Physics Letters 2004;84(21):4203-4205.

[60] Shima, H., Sato, M.. Pressure-induced structural transitions in multiwalled carbon nanotubes. physica status solidi (a) 2009;206:2228-2233.

[61] Chang, T.. Dominoes in carbon nanotubes. Phys Rev Lett 2008;101:175501.

[62] Kingston, C., Zepp, R., Andrady, A., Boverhof, D., Fehir, R., Hawkins, D., et al. Release characteristics of selected carbon nanotube polymer composites. Carbon 2014;68:33 - 57 . 